\title{
Impact des extraits éthyliques des feuilles de Hexalobus Monopétalus sur le métabolisme protéiques des rats Wistar
}

\author{
Akotegnon Rodrigue, Michodjehoun Loetitia, Behanzin Justin, Houeze Elisabeth and Sezan Alphonse \\ Laboratory of Biomembranes and Cell Signaling,University of Abomey Calavi, 06 BP 3041 Cotonou, Benin
}

Received 01 Nov 2017, Accepted 31 Dec 2017, Available online 01 Jan 2018, Vol.6 (Jan/Feb 2018 issue)

\begin{abstract}
The aim of this study was to evaluate the impact of the ethyl extract of dried leaves of Hexalobus monopetalus on protein metabolism in Wistar rats. After a phytochemical screening of extracts, total protein, urea, uric acid and creatinine were measured spectrophotometrically at six (06) sets of three (03) Wistar rats each. Batches 2 to 6 were given with orally administered respectively the daily doses of: $0.25 \mathrm{mg} / \mathrm{kg}, 0.5 \mathrm{mg} / \mathrm{kg}, 1 \mathrm{mg} / \mathrm{kg}, 1.5 \mathrm{mg} / \mathrm{kg}$ and $2.5 \mathrm{mg}$ $\mathrm{kg}$ body weight of the extracts (for 14 days). The lot1 witness received distilled water instead of the extract. The results of the phytochemical screening revealed the presence polyphenolic compounds (gallic tannins, catechin tannin or condensed, anthocyanins, and leuco anthocyanins), flavonoids, mucilages reducing compounds, alkaloids, certain anthracene derivatives ( free anthracene, the O-glycosides), Steroids, and quinone derivatives. It was observed a significant increase $(p<0.05)$ blood levels of total protein and uric acid and a significant $(p<0.05)$ blood urea and creatinine levels in treated plots to extracts. These observed variations did not depend on the administered dose of the extracts but were depending on the duration of treatment; they were outstanding from the 7th day of treatment.

The long term use of ethyl extract of dried leaves of Hexalobus monopetalus could compromise the liver function and the kidneys and may inhibit apoptosis in Wistar rats.
\end{abstract}

Keywords: Hexalobus monopetalus, protein, urea, creatinine, uric acid.

\section{Introduction}

Au fil des siècles, apprenant à distinguer le comestible du mortel, à se servir des substances toxiques aux dépens de leurs ennemis, à reconnaitre les vertus curatives cachées dans leur environnement naturel, nos ancêtres nous ont légué une longue chaîne de savoirs traditionnels dont l'ensemble constitue la médecine traditionnelle actuelle. L'expression médecine traditionnelle se rapporte aux pratiques, aux méthodes savoirs et croyances en matière de santé qui impliquent l'usage à des fins médicinales des plantes, des parties d'animaux et de minéraux, de thérapies spirituelles, de techniques et d'exercices manuels, séparément ou en association, pour soigner, diagnostiquer, et prévenir les maladies ou préserver la santé (Eddouks et al.,2007). [1]

De nos jours, bien que la médecine moderne soit développée presque partout dans le monde, une proportion non négligeable de la population dans les pays en développement compte encore sur les tradipraticiens, les plantes médicinales et les médicaments à base de plantes pour leurs soins de base (Zhang, 1998) [2]. Selon

*Corresponding author's ORCID ID: 0000-0001-5210-1565 DOI: https://doi.org/10.14741/ijmcr.v6i01.10900 les estimations de l'Organisation Mondiale de la Santé (OMS), plus de $80 \%$ de la population mondiale et surtout dans les pays sous-développés ont recours aux traitements traditionnels pour satisfaire leurs en matière de santé et de soins primaires (Eddouks et al., 2007). [1] En Afrique et particulièrement au Bénin, des études menées sur plusieurs plantes ont prouvées leurs vertus thérapeutiques dont celles de Hexalobus monopetalus.

Hexalobus monopetalus, l'une des six espèces du genre Hexalobus de la famille des Anonaceaes est originaire de l'Afrique tropicale. On la retrouve dans les pays comme le Bénin, la Tanzanie, la Côte-d'Ivoire, le Sénégal. Ses organes végétatifs sont utilisés dans dont le traitement de la céphalée et du diabète, (Akouègninou et al., 2006),[3] des candidoses (Hamisi et al., 2014)[4] etc. Dans la littérature, beaucoup de travaux $\mathrm{n}^{\prime}$ avaient pas abordé des études fondamentales sur les feuilles de cette plante. C'est dans cette optique que nous nous sommes fixé pour objectif d'évaluer l'impact de l'extrait éthylique des feuilles de Hexalobus monopetalus sur le métabolisme protéique des rats Wistar.

\subsection{Généralité sur Hexalobus Monopetalus}

1-1-1 Position systématique de Hexalobus monopetalus et noms vernaculaires (communs)

1|Int. J. of Multidisciplinary and Current research, Vol.6 (Jan/Feb 2018) 
Règne:

Végétal

Embranchement :

Classe :

Ordre :

Famille :

Genre :

Espèce :

Magnoliophyta

Magnoliopsida

Magnoliales

Annonaceae

Hexalobus

Hexalobus monopetalus
1-1-2 Description de Hexalobus monopetalus

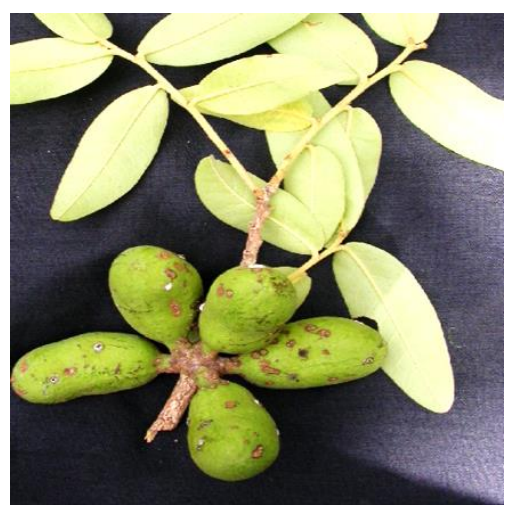

Photo 1: Les feuilles et les fruits de Hexalobus monopetalus

Le genre Hexalobus monopetalus est natif d'Afrique tropicale, sauf une espèce endémique de Madagascar. C'est un genre relativement petit sur environ six espèces de cette famille (Hexalobus callicarpus, Hexalobus crispiflorus, Hexalobus monopetalus, Hexalobus mossambicensis, Hexalobus salicifolius).

Il s'agit d'arbres ou d'arbustes persistants, aux feuilles alternes, simples et entières. Les fleurs $(F F=3 S+6 P+n E$ $+3-12 \mathrm{C}$ ), actinomorphes et bisexuées, sont solitaires ou groupées en fascicules axillaires. Elles se composent de trois (03) sépales libres, coriaces, valvaires, de six (06) pétales cornés à la base en un tube court, disposés sur un (01) seul verticille, de nombreuses étamines libres, et de 3-12 carpelles libres et pubescents, disposés sur un réceptacle convexe, et formant un ovaire supère, chacun possédant de nombreux ovules disposés sur 1-2 verticilles. Les fruits sont composés de méricarpes oblongs ou cylindriques.

\section{1-1-3 Activités biologiques de Hexalobus monopetalus}

Hexalobus monopetalus, est utilisé traditionnellement dans le traitement de plusieurs maladies: les troubles stomacaux, la fièvre et le paludisme. Le décocté aqueux des racines de cette plante en association avec les écorces du tronc de Ficus glumosa est utilisé par voie orale pour le traitement du diabète (Akouègninou et al., 2006). [3] L'huile essentielle de ses fruits est aussi utilisée pour traiter les candidoses (Hamisi et al., 2014) )[4]. La macération aqueuse de la poudre de l'écorce est utilisée au Sénégal pour traiter les maux de ventre, la fièvre jaune et la conjonctivite chez les bovins (Omar et al., 2014).[5] L'extraction de l'huile essentielle des fruits de Hexalobus monopetalus par Hamisi et al (2014) )[4] montre qu'ils contiennent plusieurs composés dont les alcaloïdes de type 3,5 hexalobine $C$ et le 3,5 hexalobine $D$ qui ont une activité antifongique contre les Candida Albicans.

\section{Matériels et Méthodes}

\section{2-1 Matériels}

\section{2-1-1 Matériel Végétal}

Le matériel végétal utilisé est constitué des feuilles de Hexalobus monopetalus, récoltées en septembre 2014 par l'équipe du Laboratoire de Biomembranes et Signalisation Cellulaire. Les feuilles étaient séchées à l'étuve à une température de $50^{\circ}$ pendant au moins $4 \mathrm{~h}$. Ces feuilles séchées ont été broyées finement. La poudre obtenue est ensuite conservée dans des bocaux de verre afin d'éviter l'installation de micro-organismes polluants.

\section{2-1-2 Matériel animal}

Le matériel animal est constitué des rats de type Wistar, soumis à un gavage sub-chronique (14 jours) et élevés à l'animalerie du Laboratoire de Biomembranes et de Signalisation Cellulaire. Ces animaux ont un accès libre à la nourriture et à l'eau.

\section{2-2 Méthodes}

2-2-1 Préparation de l'extrait éthylique des feuilles de Hexalobus monopetalus

Pour l'obtention de l'extrait éthylique des feuilles de Hexalobus monopetalus, 112,5 g de poudre obtenue, pesée à l'aide $d^{\prime}$ une balance analytique Sartorius ${ }^{\circledR}$ est macéré dans $750 \mathrm{ml}$ d'éthanol sous agitation continue pendant 72 heures. Ensuite, le macérât est filtré au travers du coton fibre hydrophile. Le filtrat obtenu est évaporé à $40^{\circ} \mathrm{C}$ à l'aide de l'évaporateur rotatitif Rotavapor $^{\circledR}$ et la maille est réutilisée pour une seconde macération afin d'augmenter le rendement. La pâte déposée au fond du ballon de l'évaporateur est récupérée dans des bocaux et mise au séchage à l'étuve à $45^{\circ} \mathrm{C}$. Après séchage complet, les extraits secs accolés au fond des bocaux sont raclés à l'aide de la spatule en inox, concassés dans le mortier de porcelaine puis gardés dans des flacons en verre préalablement étiquetés.Ce sont ces extraits qui seront utilisés pour préparer les gammes de concentration testées.

Le rendement est déterminé par le rapport du poids de l'extrait sec après évaporation sur le poids de la matière végétale sèche utilisée pour l'extraction multiplié par 100\% (Bekhechi 2001) [6] cité par Medane ,2012)).[7]

Rendement $\%=\left(m_{1} \times 100\right) / m_{2}$

$m_{1}$ : la masse de l'extrait sec après évaporation $m_{2}$ : la masse de la matière végétale sèche

2 | Int. J. of Multidisciplinary and Current research, Vol.6 (Jan/Feb 2018) 


\section{2-2-2 Screening phytochimique}

Le screening phytochimique est une analyse chimique basée sur des réactions de coloration ou de précipitation plus ou moins spécifiques à chaque classe de principes actifs. II est réalisé selon la méthode de Houghton et Raman (1998).

\section{2-2-3 Gavage des rats}

Le gavage des rats consiste à administrer les extraits éthyliques des feuilles de Hexalobus monopetalus à des rats Wistar (mâles et femelles) d'environ $173 \mathrm{~g}$ et ayant la même ration alimentaire, par voie orale et à diverses doses pendant 14 jours. Ces animaux ne reçoivent aucun autre traitement médicamenteux dans le temps en dehors de l'extrait.

Les rats ont été répartis en six (06) lots de trois rats chacun numérotés lot 1 , lot 2 , lot 3 , lot 4 , lot 5 , lot 6 . Les lots 2 à 6 reçoivent respectivement les doses de $0,25 \mathrm{mg} / \mathrm{kg}, 0,5 \mathrm{mg} / \mathrm{kg}, 1 \mathrm{mg} / \mathrm{kg}, 1,5 \mathrm{mg} / \mathrm{kg}$ et $2,5 \mathrm{mg} / \mathrm{kg}$ de poids corporel de l'extrait éthylique des feuilles de Hexalobus monopetalus puis on fixe le volume à $1 \mathrm{ml}$. Ensuite, on pèse chacun des rats de chaque lot afin de trouver le poids moyen et on calcule la dose effective d'extrait qui lui sera administrée. On gave quotidiennement et à la même heure les rats pendant 14 jours. Le lot 1 , témoin reçoit de l'eau distillée à la place de l'extrait éthylique des feuilles de Hexalobus monopetalus.

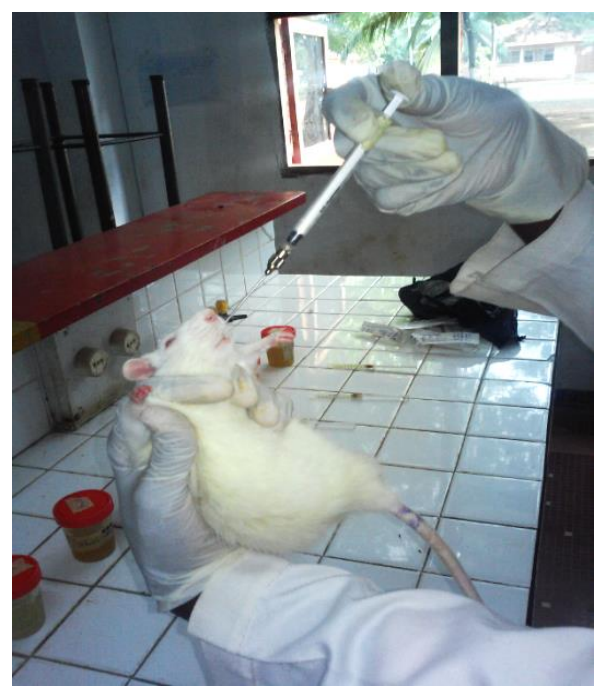

Photo 2 : Gavage des rats Wistar avec l'administration des extraits éthyliques des feuilles de Hexalobus monopetalus

\section{2-2-4 Dosage des paramètres biochimiques}

\section{a) Prélèvement sanguin}

Le prélèvement sanguin est réalisé selon le protocole expérimental employé par Weiss et al. (2000), [8] et modifié par Descat (2002)[9]. La ponction du sinus retro- orbitaire a été réalisée sous anesthésie. L'animal anesthésié est maintenu d'une main en décubitus latéral, et tenu par la peau du cou. La pression du pouce sur le cou, derrière l'angle de la mâchoire permet de réaliser une compression de la veine jugulaire, et donc une stase veineuse vers la tête, favorisant le remplissage du sinus rétro-orbitaire. En effectuant une légère traction sur la paupière supérieure avec l'index, nous créons une exophtalmie facilitant le prélèvement de sang à l'aide de tube à hématocrite non hépariné. L'extrémité du tube est introduite lentement dans l'angle latéral de l'œil. La progression à travers les tissus est facilitée en imprimant une légère rotation à la pipette. Dès qu'on atteint le plexus veineux, le sang jaillit dans l'espace périorbitaire et monte par capillarité dans le tube. Le volume de sang collecté est de 0,5 à $2 \mathrm{ml}$. Avant le retrait du tube, on relâche la compression et le saignement cesse spontanément lorsque la pression oculaire se normalise. Le sang récupéré est utilisé pour le dosage des différents paramètres biochimiques.

\section{b) Dosage des protéines totales}

\section{Principe}

Le dosage des protéines totales a été réalisé par la méthode calorimétrique décrite par Gornall et al., (1949) [10]. Les liaisons peptidiques des protéines réagissent avec les ions cuivriques $\left(\mathrm{Cu}^{2+}\right)$ en solution alcaline pour former un complexe coloré dont l'absorbance, proportionnelle à la concentration en protéines dans le spécimen, est mesurée à $550 \mathrm{~nm}$. Le réactif Biuret contient du sodium potassium tartrate qui complexe les ions cuivriques $\left(\mathrm{Cu}^{2+}\right)$ et maintient leur solubilité en solution alcaline.

\section{c) Dosage de l'urée}

\section{Principe}

La méthode utilisée est la méthode enzymatique basée sur la réaction décrite par Talke et Schubert (1895)[11] et optimisée par Tiffany et al [12]

La diminution de l'absorbance due à la conversion du $\mathrm{NADH}$ en NAD+, mesurée pendant un temps donné à 340 $\mathrm{nm}$, est proportionnelle à la concentration en urée dans le spécimen.

\section{d) Dosage de la créatinine}

\section{Principe}

La méthode utilisée est la réaction (réaction de Jaffé, sans étape de pré-traitement du spécimen) de la créatinine avec l'acide picrique en milieu alcalin dont la cinétique de développement est mesurée à $490 \mathrm{~nm}$ (490-510).

Cette méthode a été optimisée (spécificité, rapidité et adaptabilité) par le développement d'une méthode cinétique à deux (02) points.

3 | Int. J. of Multidisciplinary and Current research, Vol.6 (Jan/Feb 2018) 


\section{e) Dosage de l'acide urique}

Principe

L'uricase agit sur l'acide urique pour produire de l'allantoine, du dioxyde de carbone et du peroxyde d'hydrogène. En présence de peroxydase,le peroxyde d'hydrogène réagit avec un chromogène (dichlorohydroxybenzène sulfonate et amino-antypirine) pour former une quinoneimine, complexe de couleur rouge. L'absorbance mesurée à 520 (490-530), est proportionnelle à la quantité d'acide urique dans le spécimen.

\section{2-2-5 Analyse statistique}

L'analyse statistique est réalisée à l'aide du logiciel Minitab 14 en utilisant le test de variance univariéé (oneway ANOVA) suivie du test de Tukey.

\section{Results and Analysis}

Cette étude nous montre qu'après extraction éthylique de la poudre des feuilles de Hexalobus monopetalus, nous avons obtenu un rendement de $9 \%$.

Les résultats du screening phytochimique ont montré la présence de plusieurs composés chimiques dotés de propriétés diverses et à diverses proportions (confère tableau suivant). Il s'agit des composés polyphénoliques tels que : les tanins galliques, les tanins catéchiques ou condensés, les anthocyanes, et les leuco-anthocyanes; des flavonoïdes; des mucilages; des composés réducteurs; des alcaloïdes; de certains dérivés anthracéniques tels que: les anthracéniques libres, les 0 Hétérosides; des stéroïdes et des dérivés quinoniques. S'agissant des alcaloïdes, ils ont également été détectés dans les huiles essentielles des fruits de Hexalobus monopetalus par Hamisi et al. (2014) [4]. Ce qui suppose que ce composé ne se trouve pas uniquement au niveau des feuilles.

Tableau 1 : Résultats du screening phytochimique

\begin{tabular}{|c|c|c|}
\hline Groupes Chimiques & Sous-groupes & Observations \\
\hline \multirow{4}{*}{$\begin{array}{c}\text { Les composés poly } \\
\text { phénoliques }\end{array}$} & $\begin{array}{c}\text { Tanins } \\
\text { Tanins galliques }\end{array}$ & +++ \\
\cline { 2 - 3 } & $\begin{array}{c}\text { Tans catéchiques ou } \\
\text { condensés }\end{array}$ & ++ \\
\cline { 2 - 3 } & Anthocyane & ++ \\
\cline { 2 - 3 } & Leuco anthocyane & +++ \\
\hline Flavonoïdes & Flavone & +++ \\
\hline Mucilage & & +++ \\
\hline $\begin{array}{c}\text { Composés } \\
\text { réducteur }\end{array}$ & & +++ \\
\hline Saponosides & & ++ \\
\hline Alcaloïdes & & - \\
\hline $\begin{array}{c}\text { Dérivés } \\
\text { cyanogéniques }\end{array}$ & & +++ \\
\hline Dérivés & Anthracéniques libres & + \\
\hline
\end{tabular}

\begin{tabular}{|c|c|c|}
\hline \multirow[t]{2}{*}{ anthracéniques } & $\begin{array}{l}\text { O. Hétérosides } \\
\text { (A. combinés) }\end{array}$ & + \\
\hline & $\begin{array}{l}\text { C. Hétérosides } \\
\text { (A. combinés) }\end{array}$ & - \\
\hline Triterpènoides & & - \\
\hline Stéroides & & + \\
\hline Coumarines & & - \\
\hline $\begin{array}{l}\text { Les dérivés } \\
\text { Quinoniques }\end{array}$ & & +++ \\
\hline & $\begin{array}{c}\text { Absent } \\
\text { +Peu abondant } \\
++ \text { Abondant } \\
+++ \text { Très abondant }\end{array}$ & \\
\hline
\end{tabular}

La figure ci-dessous présente la variation de la concentration des protéines totales des différents lots de rats en fonction des doses reçues d'extrait éthylique des feuilles de Hexalobus monopetalus.

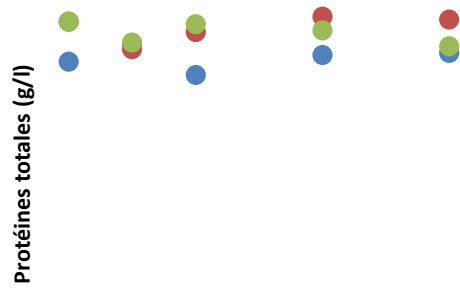

$$
\begin{gathered}
\text { Extraits ethyliques des feuilles Hexalobus } \\
\text { monopetalus }(\mathrm{mg} / \mathrm{kg} \text { de } \mathrm{pc})
\end{gathered}
$$

Figure 1: Evolution de la concentration de protéines totales en fonction des doses d'extraits éthyliques des feuilles de Hexalobus monopetalus et du temps.

L'analyse de la figure 2 nous montre que l'effet des différentes doses d'extraits éthyliques des feuilles de Hexalobus monopetalus n'est pas significativement différent pour la variation des concentrations en protéines totales $(P>0,05)$. Par contre, les différents temps d'administration de l'extrait éthylique des feuilles de Hexalobus monopetalus influencent significativement les variations des concentrations en protéines totales chez les rats Wistar $(P=0,003)$ et ceci se traduit par une élévation du taux sanguin de protéines totales le septième (7è) jour de l'administration des extraits.

Les protéines jouent un rôle dans le métabolisme cellulaire en agissant comme catalyseur des réactions chimiques, mais interviennent également dans la structure des tissus, le stockage et le transport de petites molécules et d'ions, les communications cellulaires, le mouvement par contraction des muscles et les défenses de l'organisme contre des éléments étrangers ou des cellules cancéreuses (Racano et al 2014).[13]

L'élévation de la concentration sanguine en protéines totales après administration par voie orale des extraits éthyliques des feuilles de Hexalobus monopetalus aux rats Wistar pourrait être due, soit aux stéroïdes qu'ils 4 | Int. J. of Multidisciplinary and Current research, Vol.6 (Jan/Feb 2018) 
contiennent, soit à une nécrose ou à l'inhibition de l'apoptose, soit à l'élevation du taux d'acide urique sanguin. En effet,nos résultats ont montré une élévation du taux d'acide urique après administration par voie orale des extraits éthyliques des feuilles de Hexalobus monopetalus; ce qui pourrait justifier l'élévation des protéines totales. L'urée étant un bon témoin du métabolisme protidique (Burtis et al.,1999). [14] Cette augmentation ferait aussi penser à l'inhibition du catabolisme des protéines, vu la diminution de la concentration en urée observée sur la figure suivante.

En effet cette figure présente l'évolution des différentes concentrations en urée des différents lots de rats en fonction des doses d'extraits éthyliques des feuilles de Hexalobus monopetalus et du temps.

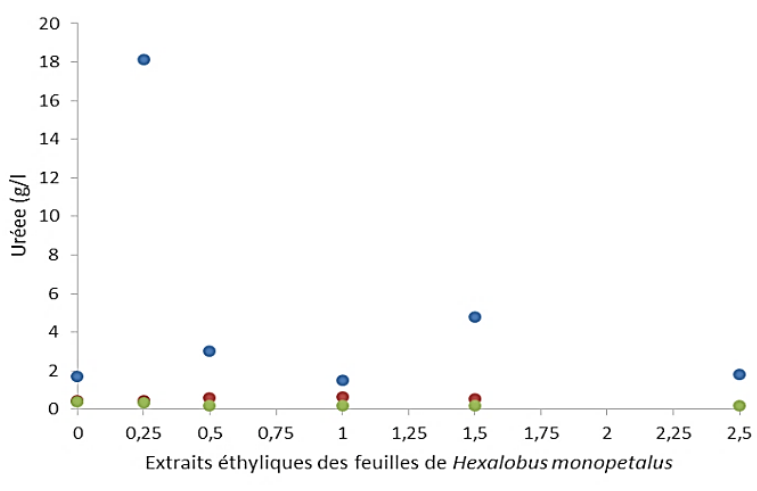

๑ Jo • Jo+7 • Jo+14

Figure 2: Evolution de la concentration en urée en fonction du temps et des doses d'extraits éthyliques des feuilles de Hexalobus monopetalus et du temps.

Après analyse de la figure 3 , on constate que ni les différents temps d'administration ni les différentes doses des extraits éthyliques des feuilles de Hexalobus monopetalus n'ont pas eu un effet significatif sur les variations des concentrations en urée $(P>0,05)$.

L'urée est la principale forme d'élimination des déchets azotés issus du métabolisme des protéines. Plus de $90 \%$ de l'urée est éliminée par les reins dans l'urine (Burtis et al., 1999) [14]. Son dosage dans le sang et/ou dans les urines explore donc essentiellement la fonction rénale. L'urée est aussi un bon témoin du métabolisme protidique (utilisation des protéines par l'organisme). Certains facteurs non rénaux influencent également sa concentration. II s'agit notamment du catabolisme accéléré des protéines provoquant une augmentation de la concentration en urée (brûlures, traumatisme, infarctus du myocarde). Par contre, le taux d'urée est abaissé au stade terminal d'une grande insuffisance hépatique et s'accompagne d'une augmentation de l'ammoniémie. (Burtis et al., 1999) [15]. Les travaux menés dans le Laboratoire de Biomembranes et de Signalisation Cellulaire ont montré que les extraits éthyliques des feuilles de Hexalobus monopetalus augmentent le taux sanguin d'ASAT qui est une enzyme fondamentale de la fonction hépatique. Cependant, la diminution de la concentration en urée observée sur la figure 3 pourrait s'expliquer soit par une insuffisance hépatique induite par l'extrait éthylique des feuilles de Hexalobus monopetalus, soit par une auto-élimination de l'urée par les rats dans le temps.

La figure ci-dessous présente l'évolution des différentes concentrations en créatinine des différents lots de rats en fonction des doses d'extraits éthyliques des feuilles de Hexalobus monopetalus et du temps.

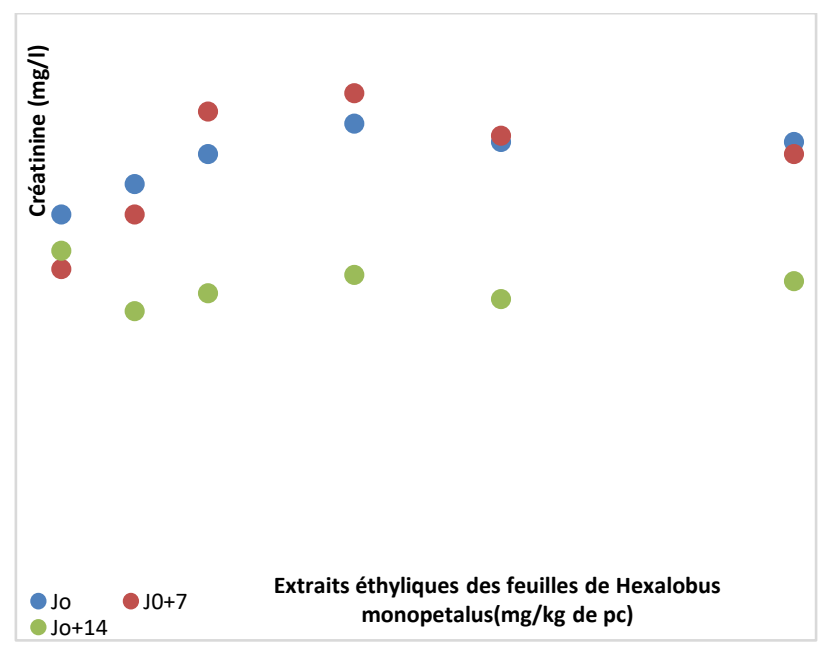

Figure 3 : Evolution de la concentration de créatinine en fonction des doses d'extraits éthyliques des feuilles de Hexalobus monopetalus.

Après analyse de la figure 4 , nous constatons que les différentes doses d'extraits éthyliques des feuilles de Hexalobus monopetalus n'ont pas eu un effet significatif sur les variations des concentrations en créatinine $(P>0,05)$. Par contre, les différents temps d'administration des extraits éthyliques des feuilles de Hexalobus monopetalus influencent significativement les concentrations en créatinine $(P<0,05)$ chez les rats Wistar, par la diminution de la concentration en créatinine. Cette diminution est prononcée le 14è jour de l'administration des extraits éthyliques des feuilles de Hexalobus monopetalus.

La créatinine est un dérivé de la dégradation de la créatine et du phosphocréatine qui sont considérés comme des composés de stockage de l'énergie dans les muscles. La concentration de la créatinine dans le sérum peut varier selon un certain nombre de facteurs dont le régime alimentaire, la masse musculaire et le sexe. Elle dépend aussi de la capacité des reins à éliminer la créatinine, d'où son utilisation aussi comme indicateur de l'insuffisance rénale (Boubchir et al., 2002) [16] cité par (Boumaza , 2009) [17]. Aussi, la concentration de la créatinine est considérée souvent comme un paramètre clinique pour détecter les effets toxiques reliés au traitement par des composés sur les reins chez les animaux expérimentaux (Travlos et al., 1996) [18] cité par (Boumaza , 2009) [17]. Une diminution significative de ce 
taux en créatinine chez les rats traités oralement par les extraits éthyliques des feuilles de Hexalobus monopetalus, nous amène à penser à la diminution du catabolisme de la créatine et la phosphocréatine (Boumaza A, 2009) [17] au niveau des muscles par le biais des flavonoïdes présents dans les extraits éthyliques de Hexalobus monopetalus. Ces résultats sont en accord avec ceux de Palsamy et al. (2008) [19] qui ont noté un effet semblable avec le resvératrole en tant que flavonoïde (Boumaza , 2009). [17]

La figure ci-dessous présente l'évolution des différentes concentrations en acide urique des différents lots de rats en fonction des doses d'extraits éthyliques des feuilles de Hexalobus monopetalus.

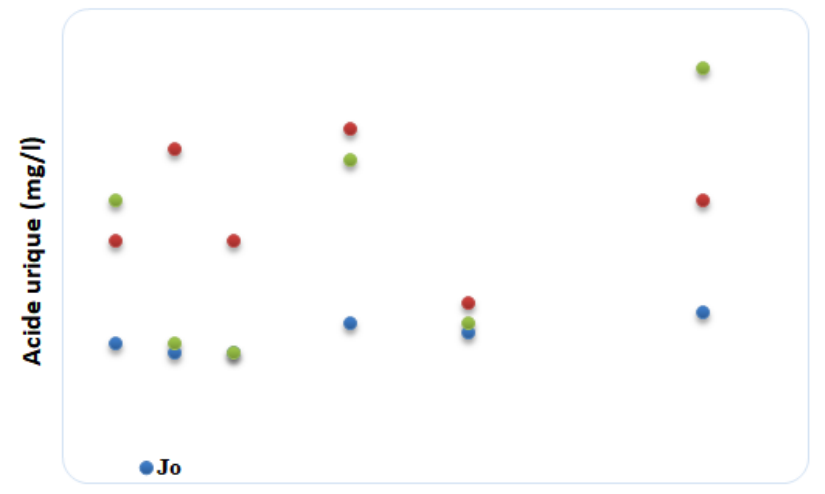

Figure 4 : Evolution de la concentration d'acide urique en fonction des doses d'extraits éthyliques des feuilles de Hexalobus monopetalus chez les lots de rats Wistar

Après analyse de la figure 5 , nous constatons que les différentes doses d'extraits éthyliques des feuilles des Hexalobus monopetalus n'ont pas eu un effet significatif sur les concentrations en acide urique $(P>0,05)$ tandis que les différents temps influencent significativement les concentrations en acide urique $(P<0,05)$ chez les rats Wistar. Cette influence se traduit par une élévation de la concentration en acide urique qui se prononce à partir du 7è jour de l'administration de l'extrait éthylique des feuilles de Hexalobus monopetalus.

L'acide urique, produit ultime du catabolisme des bases puriques (Boubchir, 2002) [16] cité par (Boumaza, 2009) [17] est l'un des antioxydants hydrosolubles endogènes majeurs de l'organisme (Delattre et al., 2005) [20] cité par (Boumaza A.,2009) [17]. Dans notre étude, nous avons constaté l'augmentation significative $(p<$ 0.05) du taux de l'acide urique à partir du 7è jour de l'administration des extraits éthyliques des feuilles de Hexalobus monopetalus. D'après Modan et al. (1987) [21], cette augmentation peut être due, soit à la production accélérée de l'acide urique, soit à la diminution de sa sécrétion (Boumaza., 2009). [17] Le taux élevé de l'acide urique circulant peut être un indicateur de défense de l'organisme contre les effets délétères des radicaux libres dont il augmente la production des antioxydants endogènes parmi lesquels l'acide urique qui prévient les modifications oxydatives des enzymes endothéliales et préserve la capacité de l'endothélium à méditer des dilatations vasculaires pour faire face au stress oxydant (Becker et al., 1993) [22] cité par (Boumaza ,2009) [17] . Dans la biologie clinique, une concentration sanguine élevée de l'acide urique est considérée comme indicateur pathologique reflétant une insuffisance rénale (Boubchir et al., 2002) [16] cité par (Boumaza, 2009) [17]. Cette élévation peut aussi être induite par l'augmentation de la concentration des triglycérides, du faible taux de cholestérol HDL et l'augmentation de la glycémie observés au cours des travaux effectués sur la même plante dans le Laboratoire de Biomembranes et de Signalisation Cellulaire.

En résumé nous pouvons dire que les extraits éthyliques des feuilles de Hexalobus monopetalus induisent une élévation des concentrations sanguines des protéines totales et de l'acide urique puis une diminution des concentrations sanguines de la créatinine chez le rat Wistar.

\section{Conclusion}

Les feuilles de Hexalobus monopetalus sont constituées de quatre (04) sous-classes de composés polyphénoliques (les tanins galliques, les tanins catéchiques ou condensés, les anthocyanes, et les leuco-anthocyanes), de flavonoïdes, de mucilages, de composés réducteurs, d'alcaloïdes, de deux (02) types de dérivés anthracéniques (les anthracéniques libres, les 0 hétérosides), de stéroïdes, et de dérivés quinoniques.

L'administration par voie orale des extraits éthyliques des feuilles séchées de Hexalobus monopetalus pendant 14jours chez le rat wistar a provoqué la diminution des concentrations sanguines en urée et en créatinine puis l'augmentation des concentrations sanguines en protéines totales et en acide urique. Les variations observées dans ces paramètres biochimiques étudiés est fonction de la durée du traitement et ne sont pas liées à la dose administrée.

L'usage systémique prolongé des extraits éthyliques des feuilles de Hexalobus monopetalus pourraient causer un dysfonctionnenent du foie et des reins ou inhiber l'apoptose

\section{References}

[1] M.Eddouks; M.L.Ouahidi.O.Farid, A.Moufid,A.Khalidi, A.Lemhadri(2007) L'utilisation des plantes médicinales dans le traitement du diabète au Maroc, Physiologie et Pharmacologie Endocrinienne,Faculté des Sciences et Techniques Errachidia,Phytothérapie, vol: (5) pp194-203.Ed Springer2007

[2] Zhang X. (1998) Réglementation des médicaments à base de plante : La situation dans le monde.WHO/TRM. 65p

[3] Akouègninou A., Vander Burg W.J et Vander Maesen L.J.G. (2006) Flore analytique du Bénin. Brackhuys publishers Wageningen, $1034 \mathrm{p}$.

[4] Hamisi M. M., Stephan A. J., Reiner W., and Mayunga H. H. N. (2014) Diprenylated Indole Alkaloids from Fruits of Hexalobus monopetalus, Natural Products and Bioprospecting. pp101-105

6 | Int. J. of Multidisciplinary and Current research, Vol.6 (Jan/Feb 2018) 
[5] Omar N. G .et al. (2014) Richesse de la pharmacopée malinké: Rôle médicinal de l'arbre à Khossanto (Kédougou, Sénégal oriental)

[6] Bekhechi-Benhabib, C., (2001) Analyse d'huile essentielle d'Amoïdes verticillata (Nûnkha) de la region Tlemcen et étude de son pouvoir antimocrobien. Thèse de magister de Biologie, Université Abou Bekr Belkaid de Tlemcen. Algérie.

[7] Medane A. (2012) Evolution des paramètres biochimiques sériques chez les rats wistar traités par l'extrait chloroformique des graines de la coloquinte Citrullus colocynthis. Mémoire en vue de l'obtention du Diplôme de Master en biologie. Université Abou Bekr Belkaid - Tlemcen.Algérie.66p

[8] Gornall A.C., Bardawill C.J.,David M.M. J.(1 949 ) Biol Chem pp 117-751

[9] Talke H.Schubert G.E.(1695) Klin. Wochschr, p. 174

[10] Tyffany T.O. et al , (1972) Clin Chem 18 pp 829-840

[11] Racano S., Rigo P., (2014) Biologie cellulaire: exercices et méthodes. Ed Dunod, Paris, France $.28 p$
[12] Burtis C.A., Ashwood E.R., Saunders W.B. (1999) Tietz N.W Text book of clinical chemistry ,3rd Ed pp1245-1250

[13] Boubchir M.A. (2002) Biochimie de néphrologie. 2ème Ed. ISBN-00-789-23:320

[14] Boumaza A. (2009) Effet de l'extrait méthanolique de Zygophyllum cornutum coss contre le stress oxydant associé au diabète sucré et les organes en relation. Mémoire En vue de l'obtention du Diplôme de Magister en Biologie cellulaire et moléculaire Option : Toxicologie cellulaire et moléculaire.126p

[15] Travlos G.S., Morris R.W., Elwell M.R. Duke A., Rosenblum S., Thompson M.B. (1996) Frequency and relationships of clinical chemistry and liver and kidney histopathology findings in 13week toxicity studies in rats.Toxicology; 107:17-29

[16] Palsamy P. and Subramanian S. (2008) Resveratrol, a natural phytoalexin, Normalizes hyperglycemia in STZnicotinamide induced experimental diabetic rats biomedicine pharmacotheraphy.62:pp598-605

[17] Delattre J. (2005) Radicaux libres et stress oxydant. Ed: TECDOC. Londres-paris -new york.620p

[18] Becker B.F. (1993) Towards the physiological function of uric acid. Free Radic boil Med; 14:615-13. 